\title{
A Compressive Sensing based BEEIP Protocol for WANETS
}

\author{
Gaganjot Kaur \\ MTech Scholar \\ Department of Computer Science \& \\ Engineering, Amritsar College of Engineering \& \\ Technology, Amritsar
}

\author{
Sandeep Kad \\ Associate Professor \\ Department of Computer Science \& \\ Engineering, Amritsar College of Engineering \& \\ Technology,
}

\begin{abstract}
Routing Algorithms in the wireless environment are differentiating into different kinds like Geographical, Geo-casting, Hierarchical, Multi-path, Power-aware, and Hybrid routing algorithms. The typical objective of this paper is to explore Swarm Intelligence based routing protocols especially Bee-Inspired based routing protocols for providing multipath routing in Wireless ad hoc networks (WANETs). WANETs influence an agent-based routing protocol that defines a number of rules including that the majority of the participating nodes follow. Using routing technique, nodes are interconnected jointly so as to reduce computational and resource costs. Swarm Intelligence uses agent-like entities from insect's societies becoming a metaphor to fix the routing problem. Various insects interchange details based on their activities been performed along with the surroundings in which they operate to ensure to perform their tasks within an adaptive, efficient and scalable manner. It has been observed that the Bee-Inspired routing has not yet used compression algorithm to apply the bandwidth in more proficient manner. Therefore this paper proposes a LCBEEIP protocol who has utilized BEEIP protocol along with the feature of Loss Less data compression. The experimental results in the proposed technique have clearly shown that the proposed technique outperforms over the available techniques.
\end{abstract}

\section{Keywords}

WANETs, BEEIP, Lossy Compression, Lossless Compression

\section{INTRODUCTION}

MANETS are illustrated as the wireless communication network through which nodes aren't in the direct range of each other. They individually establish their communication when using the different nodes to send the information in between them. They are going to occur without having fixed infrastructure and even come under the multi-hop wireless networking. Mobile ad-hoc sites can run in an exceedingly standalone style or may be very well placed with a larger network much like the Internet. Mobile ad-hoc sites can modify the desire of getting connected "everywhere and as long as they want" into reality. The mobile nodes which are often in wireless choice from the other can directly have a wireless medium to speak with one another.

\subsection{Applications of manets}

MANETS are typically used up while in the situations where non movable networks can be a lot expensive or they can be impractical in nature. There are accustomed in large number of applications for instance. [28]

\subsubsection{Personal Area Network (Pans)}

PANs are specifically intended for individual users. They can be based on various kinds of mobile devices. The chief role of PANs is usually to build an embedded network by combining some nodes which can be inside or near our bodies to change digital data between them. The interaction between nodes of numerous PANs can take full advantage of with all the facilities furnished by ad hoc network for example a health care provider can provide various lists of sensors on the patient's body to guarantee they will able to get the medical information also named as Wireless Body Area Network which is loaded with lots of applications in medical field. [28]

\subsubsection{Military Applications}

In the community of battle field military applications could be as the most popular application putting on ad hoc network because of providing a non infrastructure network which provides authentic communication and quickly failure recovery. Within the battle field, it isn't so complicated to formulate a set network for military communications.

\section{THE BEEIP ROUTING PROTOCOL}

This protocol can be a new routing protocol inspired by honeybee depending on the collaborative behaviors of honeybee foragers [31]. This routing protocol is utilized whenever information is being shared between the nodes. The main motive in this BEEIP routing protocol is usually to monitor and assess the performance from the discovered paths and choose the optimal one according to selection mechanism. It expresses the ability of honeybees to do the foraging and communicate against each other in the hive, to get efficient and productive recruitment. This BEEIP routing protocol is actually used to find multiple paths between the sources as well as destinations and in addition distribute the traffic across them in a scalable, robust and efficient manner.

\subsection{Adaptive scouting}

Whenever there is a necessity of a route between the source and the destination, a scout packet is created and sent by broadcast to the whole of the network. This scout packet accounts for finding uncommitted route to its destination [31]. If the acquiring node is definitely the required terminal, an ack_scout packet is established and sent back to the source node to ensure the successful path. A destination node is ready to create multiple ack_scout, dependant on a no of scouts it receives within the network, allowing multiple paths for being discovered. 


\subsection{Adaptive foraging}

The scouting process is taken successful when a variety of ack_scout is received by the source node. BEEIP concentrates on monitoring and constantly evaluating multiple paths [31]. Every time a forager is received by the destination node, it transfers piggybacked data towards the transport layer and converts in an ack_forager. Including the true honeybees which spend some time around the flower to get pollen or perhaps the nectar, the ack_forager stays within the destination node until some data packets will get back to the original source. While travelling home, it collects up-to-date information through the nodes it visits and all the links between them. This enables it to watch the general path's quality and can report the finding back towards the source.

\section{COMPRESSION TECHNIQUES}

Transmission of compressed information is often constructed from two processes: the sender process as well as receiver process. Sender process's duty is to initially compress the final results after which sent it via the entire communication network. On the other side, the receiver process receives the compressed data and decompresses it. Data compression algorithms are used to minimize the length of information so that it takes minimum disk space for storage and a lesser amount of bandwidth for getting transmitted with the help of a data communication channel. Moreover image compression process involves the following: [32]

1. Mapper to convert input data into a suitable form for compression to minimize the spatial redundancy exists on input image.

2. Quantizer to minimize the accuracy of mapper's output.

3. Entropy encoding to minimize the quantizer's output bits.

\subsection{Data compression methods}

Data compression algorithms may be characterized into two different parts named as:

a. Lossy Methods

b. Lossless Methods

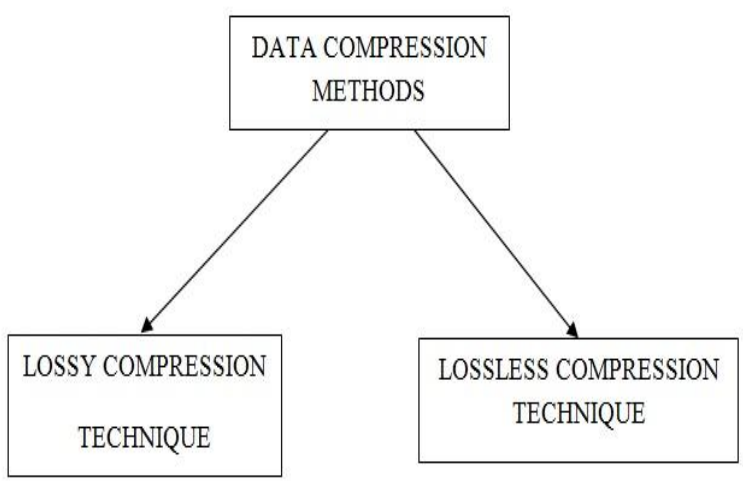

Fig 1: Data compression methods

\subsubsection{Lossy methods}

In Lossy Compression the compressed image is just not usually exactly like the original one, but forms an end approximation to the original image. Therefore, a version of a distortion measure is required. Distortion measure can be a mathematical quantity that specifies how close an approximation to the original image. In image compression the most commonly used distortion measure is MSE and PSNR.

\subsubsection{Lossless compression}

In lossless data compression, the quality of the data is conserved. The primary data and the data after compression and decompression are an identical because, within these methods, the compression and decompression algorithms are reciprocal of each other: information is disoriented in the process. Lossless compression methods are generally used up where we don't want to suffer our information. The most common type of lossless compression is Lempel $\mathrm{Ziv}$ Encoding.Lempel Ziv (LZ) encoding is a good example of a form of algorithms called dictionary-based encoding. Here, we form a table of strings used over the network. If the sender and the receiver both having the duplicate of the dictionary, then early-encountered strings may be changed by their index in the dictionary to slow down the total level of data transmitted. This encoding technique is divided into two phases i.e. Compression and Decompression.

In the compression phase you will find two coinciding events: building an indexed dictionary and compressing a string of symbols. The algorithm take out the tiniest substring that can't be exists in the dictionary from the left over uncompressed string. After so it stores a duplicate of the substring in the dictionary as a fresh entry and appoints it an index value. Compression takes place when the substring, except the past character, is exchanged with the index lies in the dictionary. The process then attaches the index and the past character of the substring into the compressed string. The Decompression process is exactly the reciprocal of the compression process. The procedure takes out the substrings from the compressed string and seeks to preempt the indexes with the relating entry in the dictionary that is discharge in the beginning and accumulated gradually. The theory is that when an index is acceptable, there is already an access in the dictionary relating to that index.

\section{LITERATURE SURVEY}

Shukla, et al. [1] illustrates some important algorithms employed for lossless compression of images. These algorithms are broadly classified as prediction based methods and transform based methods. The main element objective of this work is to provide an in depth analysis of such algorithms and to provide future research direction on the basis of the analysis to the newest researchers. Gautam, et al. [2] proposed the Swarm Routing Protocol for Mobile Ad Hoc Networks. The most up-to-date on demand ad hoc routing protocol often known as SWARM, used within the mechanisms of swarm intelligence to choose good routes to destinations. SWARM was Scalable, Adaptable, and Autonomous and possesses nice Fault tolerance capability. It provides the simulated performance comparison of SWARM with AODV and DSR in various CBR traffic flow. Performance evaluation of AODV, DSR and SWARM 
was illustrated for the behalf of packet delivery ratio, network throughput, end-to-end latency and packet transmission rate. SWARM performs greater than others. Zhang, et al. [10] explores LZW algorithm to compress the bridge vibrations data with the help of a whole new technique called forward-moving which can be applied to frequently-used entries. Simulations results indicate that this technique can effectively boost the searching speed and save compression time. Also when it is implemented by $\mathrm{C}$ language this algorithm is turned out to be own high compression efficiency with a shorter time cost in compressing bridge vibration data. Asaju la ARO Bolaji, et al. [23] analyzes the $\mathrm{ABC}$ algorithm i.e. Artificial Bee Colony Algorithm (ABC) that's nature-inspired Meta heuristic technique which simulate the foraging behavior of bees. $\mathrm{ABC}$ is tailored successfully, to solve a large collection of discrete and continuous optimization problems. They gives a complete and extensive overview coming from all research work being focused on the employment of $\mathrm{ABC}$, in return for they want it would be the reference material to both old and new, incoming researchers towards field, to support their understanding of brand new trends and assist their future research results and directions. Advantages, applications and drawbacks of the newly developed $\mathrm{ABC}$ hybrids are highlighted, critically analyzed and discussed accordingly. Aarti, et al. [24] analyzes mobile ad-hoc network and its particular characteristics, challenges, application, security goals and different type's security attacks at different layers. Mobile ad hoc networks (MANETs) are non-infrastructure, dynamic network made up of accumulation wireless mobile nodes that communicate together without the use of any centralized authority. Simply because of its fundamental characteristics, such as wireless medium, dynamic topology, distributed cooperation, MANETs is in danger of different kinds of security attacks like worm hole, black hole, rushing attack etc. Yahya Tashtoush a, et al. [28] proposed the Fibonacci Multipath Load Balancing protocol (FMLB) for Mobile Ad Hoc Networks (MANETs). The FMLB protocol divide transmitted packets over multiple paths through mobile nodes using Fibonacci sequence. This distribution can promote the delivery ratio as it decreases the congestion. The FMLB protocol's responsibility is always to balance the packets transmission in the chosen paths and ordering them reported by hops count. The shortest path must be used with greater regularity as opposed to other ones. The simulation results show the FMLB protocol has achieved an enhancement on packet delivery ratio, around $21 \%$, in comparison to the Ad Hoc On-demand Distance Vector routing protocol (AODV) protocol, or longer to $11 \%$ in the linear Multiple-path routing protocol. Also the actual end result shows the results of nodes pause serious amounts of speed on all the data delivery ratio and Endto-End (E2E) delay transmission time. Finally, the simulation outcomes are obtained because of the wellknown Glomosim Simulator, version 2.03, without distance or location measurements devices. Zain Eldin, et al. [32] did a survey on image compression algorithms in wireless multimedia sensor network. It has been proved that WMSN faces an problem regarding the character and the size of data being transmitted, memory resources, the energy consumed per each node for processing and transmission. The effective solution to these problems is image compression because the image contains massive degree of redundancies resulting from high correlation between pixels. The key objective with this particular survey is to review and analyze relevant research directions and the newest algorithms of image compression over WMSN. Alexandros Giagkos, et al. [31] explores the Swarm Intelligence based routing protocols, additionally together with the newly proposed bee-inspired routing protocol for attaining multi-path routing in wireless ad hoc networks of mobile nodes. Simulation results shows that the applying Swarm Intelligence provides a large amount of adaptability and efficiency that, under various network conditions, encourage the protocol to outperform traditional approaches. Zuo, et al. [36] proposed a much better Medical Image Compression Technique with Lossless compression. To be sure hospitals and medical centers produce a significant quantity of digital medical images each day, which are used for different purposes for instance surgical and diagnostic plans. Be simple storing and transmission of digital medical images is often a boon to patients and medical professionals. Because the large higher level of images, image compression must reduce steadily the redundancies in image and represents it in shorter manner for efficient archiving and transmission of images. However, compressing digital medical images since the region interesting for diagnosis is by and large small comparing to the overall image. Lossless compression techniques compress without loss from a information but have low compression rate, and lossy compression techniques can compress at high compression ratio although with a slight loss in data. Using lossless associated with medical image doesn't give enough advantage in transmission and storage and lossy techniques may lose crucial data necessary for diagnosis. In this particular paper, a much better medical image compression technique predicated on region interesting (ROI) is proposed to maximize compression. The image is firstly divided into two parts: ROI regions and non-ROI regions. Lossless compression algorithm will then be put on the marked element of ROI, and image restoration technique and the wavelet-based lossy compression algorithm are utilized to another perhaps the image. Finally, a few experiments are created to evaluate the potency of your proposed compression method.

\section{METHODOLOGY}

1. Firstly, the initialization of sensor nodes done randomly and then apply the compression on the sensed signals individually.

2. Now apply adaptive scouting in which a scout packet is generated and sent by broadcast to the rest of the network. This scout packet is responsible for discovering available path to its destination.

3. Now, adaptive foraging is applied in which the ack-forager stays at the destination node until some data packets needs to go back to initial source. After adaptive foraging, selection of optimal path is done based on speed, transmission delay etc.

4. After that detection of path failure is done followed by the decompression sensing finally the process ends. 


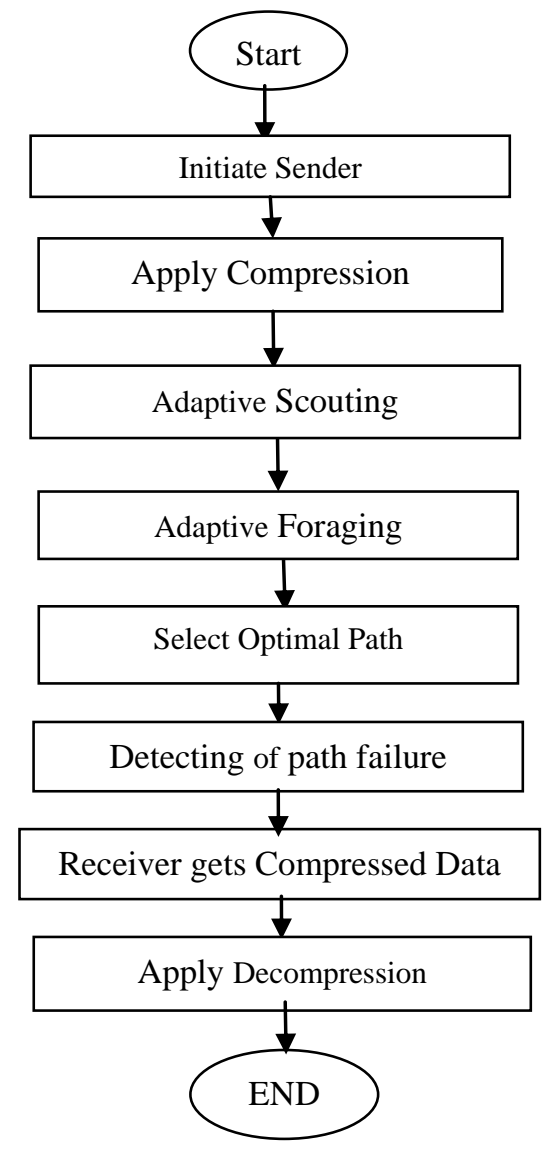

Fig 2: Flowchart of the proposed technique

\section{RESULTS}

This paper has designed and implemented the proposed technique in MATLAB tool u2013a. Table 1 shows the results of different parameters i.e. control overhead, throughput, packet delivery ratio, and end-to-end delay, based on the number of nodes which varies from 100 to 1000 .

Table1. Result Analysis of Different parameters

\begin{tabular}{|c|c|c|c|c|}
\hline $\begin{array}{c}\text { No of } \\
\text { Nodes }\end{array}$ & Throughput & $\begin{array}{c}\text { End to } \\
\text { End } \\
\text { Delay }\end{array}$ & $\begin{array}{c}\text { Control } \\
\text { overhead }\end{array}$ & PDR \\
\hline 100 & 120 & 0.0226 & 14584 & 99.1736 \\
\hline 200 & 240 & 0.0361 & 16201 & 99.5851 \\
\hline 300 & 360 & 0.0509 & 17907 & 99.7230 \\
\hline 400 & 480 & 0.0645 & 19337 & 99.7921 \\
\hline 500 & 600 & 0.0820 & 21524 & 99.8336 \\
\hline 600 & 720 & 0.1068 & 22725 & 99.8613 \\
\hline 700 & 840 & 0.1230 & 24312 & 99.8811 \\
\hline 800 & 960 & 0.1424 & 27593 & 99.8959 \\
\hline 900 & 1080 & 0.1619 & 28279 & 99.9075 \\
\hline 1000 & 1200 & 0.1830 & 31413 & 99.9167 \\
\hline
\end{tabular}

\subsection{Control overhead}

Control Overhead can be expressed as any blend of excess or indirect computation time, memory, bandwidth, or other resources that are needed to attain a certain goal. Control Overhead also describes the number of processing time installing a certain feature will add to the amount already necessary for the program.

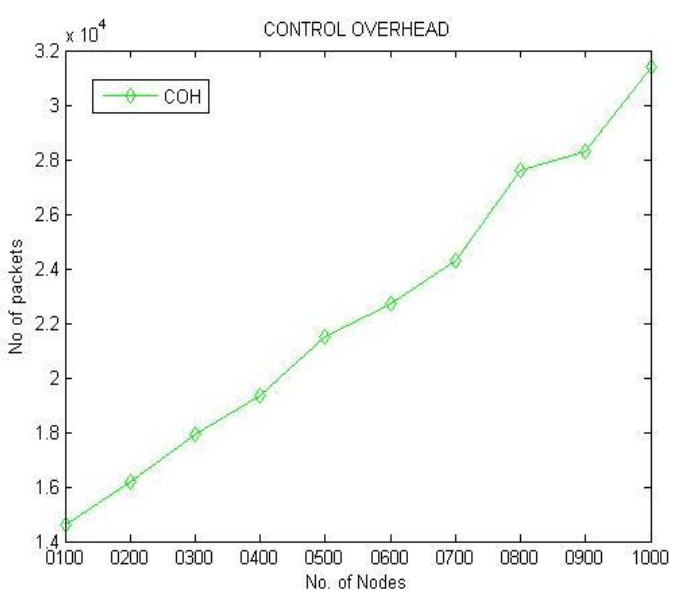

Fig 3: Control overhead ( time in seconds)

In the above fig.3 it shows the proposed Control Overhead where $\mathrm{x}$-axis represents the no of nodes and $\mathrm{y}$ axis represents the No of packets.

\subsection{End -to-end delay}

End-to-End delay generally describes the normal time taken for a packet to be transmitted across a network from source to destination. The result packet that successfully transported to destinations that matter, the lower valuation on end to end delay means the more effective performance.

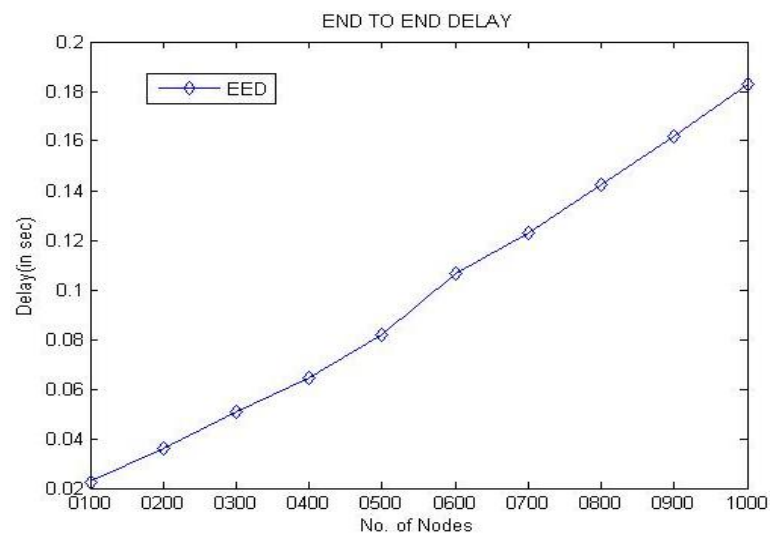

Fig 4: End to end delay (time in seconds)

In the above fig. 4 it shows the proposed End to End Delay where $\mathrm{x}$-axis represents the no of nodes and $\mathrm{y}$-axis represents the Delay (in sec).

\subsection{Throughput}

Throughput is the term which describes the pace of production or the pace in which something may be processed. In communication networks, for example Ethernet or packet radio, throughput is the pace of successful message delivery spanning a communication channel. 


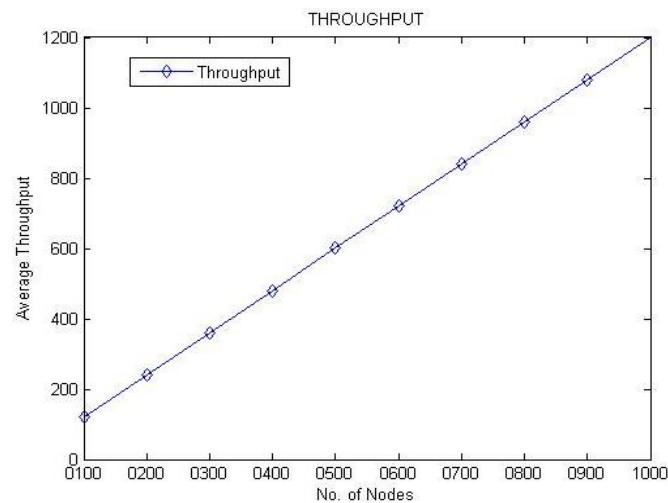

Fig 5: Throughput (packets per unit time)

In the above fig. 5 it shows the proposed Throughput where $\mathrm{x}$-axis represents the no of nodes and $\mathrm{y}$-axis represents the Average Throughput.

\subsection{Packet delivery ratio}

PDR could be thought of as the ratio of the quantity of delivered data packet towards destination. The great importance of packet delivery ratio means the better performance. It is identified as the $\sum$ Range of packet receive / $\sum$ Range of packet send.

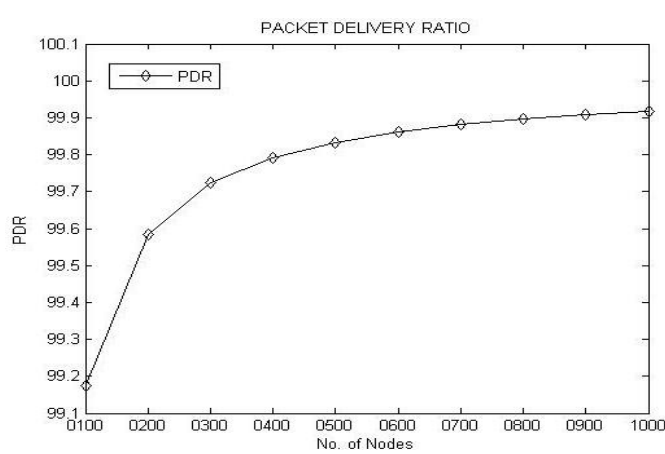

Fig 6: Packet delivery ratio $(\%)$

In the above fig.6 it shows the proposed Packet Delivery Ratio where $\mathrm{x}$-axis represents the no of nodes and $y$-axis represents the PDR.

\section{CONCLUSION}

The overall objectives of mobile ad hoc network are going to exchange a packet between the two nodes until it attain its destination. But the size of the packet may reduce the performance of protocols which are designed for mobile ad hoc networks. The size of the packet is determined by the specific application of MANETs. But high size data packets cause bad PDR, End-to-End delay and throughput on the network. In addition, it improves the overheads because each node is responsible for getting and forwarding that particular packet. This paper has devoted to Swarm Intelligence based routing protocols especially Bee-Inspired routing protocol for providing multipath routing in MANETs. But it has been observed that the Bee-Inspired routing has never used compression algorithm to utilize the bandwidth in more proficient manner. So, the LCBEEIP protocol is proposed which has utilized BEEIP protocol with the feature of Loss Less data compression. The experiments have also been done using the scalability of number of nodes. The experiments have clearly shown the effectiveness of the proposed technique over the others.

\section{REFERENCES}

[1] Shukla, Jaya, Manoj Alwani, and Anil Kumar Tiwari. "A survey on lossless image compression methods." 2010 2nd International Conference on Computer Engineering and Technology. 2010.

[2] Gautam, Sumanlata, "Swarm Routing Protocol for Mobile Ad Hoc Networks", Advances in Computing, Control and Telecommunication Technologies (ACT), 2010 Second International Conference on. IEEE, 2010

[3] Villalba, LJ García, Delfín Rupérez Cañas, and Ana Lucila Sandoval Orozco, "Bio- inspired routing protocol for mobile ad hoc networks." IET communications4.18 (2010): 2187-2195

[4] Mohseni, Shima, et al. "Comparative review study of reactive and proactive routing protocols in sMANETs." Digital Ecosystems and Technologies (DEST), 2010 4th IEEE International Conference on. IEEE, 2010.

[5] Rahman, Md Arafatur, et al. "A simulation based performance comparison of routing protocol on Mobile Ad-hoc Network (proactive, reactive and hybrid)."Computer and Communication Engineering (ICCCE), 2010 International Conference on. IEEE, 2010.

[6] Dengiz, Orhan, Abdullah Konak, and Alice E. Smith. "Connectivity management in mobile ad hoc networks using particle swarm optimization." Ad Hoc Networks 9.7 (2011): 1312-1326.

[7] Saleem, Muhammad, Gianni A. Di Caro, and Muddassar Farooq, "Swarm intelligence based routing protocol for wireless sensor networks: Survey and future directions." Information Sciences 181.20 (2011): 4597-4624.

[8] Ali, Zulfiqar, and Waseem Shahzad. "Critical analysis of swarm intelligence based routing protocols in adhoc and sensor wireless networks." In Computer Networks and Information Technology (ICCNIT), 2011 International Conference on, pp. 287-292. IEEE, 2011.

[9] Chandrasekhar, U., and P. R. P. Naga. "Recent trends in ant colony optimization and data clustering: A brief survey." In Intelligent Agent and MultiAgent Systems (IAMA), 2011 2nd International Conference on, pp. 32-36. IEEE, 2011

[10] Zhang, Fengyuan, et al. "Implementation and optimization of LZW compression algorithm based on bridge vibration data." Procedia Engineering 15 (2011): 1570-1574.

[11] Poongkuzhali, T., V. Bharathi, and P. Vijayakumar. "An optimized power reactive routing based on AODV protocol for Mobile Ad-hoc network." Recent Trends in Information Technology (ICRTIT), 2011 International Conference on. IEEE, 2011.

[12] Tan, Swee Chuan, Kai Ming Ting, and Shyh Wei Teng. "Simplifying and improving ant-based clustering." Procedia Computer Science 4 (2011): 46-55. 
[13] Alhasan, Waseem M., et al. "LDW Mean PSO: A new improved particle swarm optimization technique." Computer Engineering Conference (ICENCO), 2011 Seventh International. IEEE, 2011.

[14] Adamu Murtala Zungeru, Li-Minn Ang, Kah Phooi Seng, "Classical and swarm intelligence based routing protocols for wireless sensor networks: A survey and comparison," Journal of Network and Computer Applications 35, (2012): 1508-1536.

[15] Dhivya, M., and M. Sundarambal. "Lifetime Maximization in Wireless Sensor Networks using Tabu Swarm Optimization." Procedia Engineering 38 (2012): 511-516.

[16] Mahmudimanesh, Mohammadreza, Abdelmajid Khelil, and Neeraj Suri. "Balanced spatio-temporal compressive sensing for multi-hop wireless sensor networks." Mobile Adhoc and Sensor Systems (MASS), 2012 IEEE 9th International Conference on. IEEE, 2012

[17] Sivakumar, D., B. Suseela, and R. Varadharajan. "A survey of routing algorithms for MANET." In Advances in Engineering, Science and Management (ICAESM), 2012 International Conference on, pp. 625-640. IEEE, 2012.

[18] Santhiya, K. G., and N. Arumugam. "A novel adaptive bio-inspired clustered routing for MANET." Procedia Engineering 30 (2012): 711717.

[19] Kumar, Deepak, Ashutosh Srivastava, and S. C. Gupta. "Performance comparison of pro-active and reactive routing protocols for MANET."Computing, Communication and Applications (ICCCA), 2012 International Conference on. IEEE, 2012.

[20] Ismail, Nurul Halimatul Asmak, and Rosilah Hassan. "6LoWPAN local repair using bio inspired artificial bee colony routing protocol." Procedia Technology 11 (2013): 281-287.

[21] Sharma, Dhirendra Kumar, Chanchal Kumar, and Srimanta Mandal. "An efficient cluster based routing protocol for MANET." Advance Computing Conference (IACC), 2013 IEEE 3rd International. IEEE, 2013.

[22] Rufai, Awwal Mohammed, Gholamreza Anbarjafari, and Hasan Demirel. "Lossy medical image compression using Huffman coding and singular value decomposition." Signal Processing and Communications Applications Conference (SIU), 2013 21st. IEEE, 2013.

[23] Asaju la ARO Bolaji, Ahamad Tajudin Khader, Mohammed Azmi al-Betar and Mohammed A. Awadallah, "Artificial Bee Colony Algorithm, Its Variants and Applications: A Survey", Journal of Theoretical and Applied Information Technology, Volume 47, 2013: 434-459.

[24] Aarti, Dr S.S Tyagi, "Study of MANET: Characteristics, Challenges, Application and Security Attacks", International Journal of Advanced Research in Computer Science and Software Engineering,Volume 3, Issue 5, May 2013,pp 252-257.
[25] Kiatwuthiamorn, Jiraporn, and Arit Thammano. "A Novel Optimization Algorithm based on the Natural Behavior of the Ant Colonies." Procedia Computer Science 20 (2013): 90-95.

[26] Nandi, Utpal, and Jyotsna Kumar Mandal. "Modified compression techniques based on optimality of LZW code (MOLZW)." Procedia Technology 10 (2013): 949-956.

[27] Singh, Gurpreet, Neeraj Kumar, and Anil Kumar Verma, "ANTALG: An Innovative ACO based Routing Algorithm for MANETs." Journal of Network and Computer Applications 45 (2014): 151167.

[28] Yahya Tashtoush a,*, Omar Darwish a, Mohammad Hayajneh b, "Fibonacci Sequence Based Multipath Load Balancing Approach for mobile ad hoc network", journal, 2014

[29] Gupta, Kunal, Mukesh Sharma, and Parmanand Sharma. "Lossless compression based Kmp technique." Optimization, Reliabilty, and Information Technology (ICROIT), 2014 International Conference on. IEEE, 2014.

[30] Ramamoorthy, H. Vignesh, and H. Karthikeyani. "Hybrid routing scheme of multi agent ant based system in MANET combination of proactive and reactive." Information Communication and Embedded Systems (ICICES), 2014 International Conference on. IEEE, 2014.

[31] Alexandros Giagkos, and Myra S. Wilson, "BEEIP Swarm Intelligence based routing for wireless ad hoc networks", Information Sciences 265 (2014): 23-35.

[32] ZainEldin, Hanaa, Mostafa A. Elhosseini, and Hesham A. Ali. "Image compression algorithms in wireless multimedia sensor networks: A survey." Ain Shams Engineering Journal (2014).

[33] John, Jomy, and R. Pushpalakshmi. "A reliable optimized clustering in MANET using Ant Colony algorithm." Communications and Signal Processing (ICCSP), 2014 International Conference on. IEEE, 2014.

[34] Vanthana, S., and V. Prakash. "Comparative study of proactive and reactive adhoc routing protocols using NS2." Computing and Communication Technologies (WCCCT), 2014 World Congress on. IEEE, 2014.

[35] Jiang, Weichang, Yating Zhang, and Ruihua Wang. "Comparative study on several PSO algorithms." Control and Decision Conference (2014 CCDC), The 26th Chinese. IEEE, 2014.

[36] Zuo, Zhiyong, et al. "An Improved Medical Image Compression Technique with Lossless Region of Interest." Optik-International Journal for Light and Electron Optics (2015).

[37] Chłopkowski, Marek, and Rafał Walkowiak. "A general purpose lossless data compression method for GPU." Journal of Parallel and Distributed Computing75 (2015): 40-52. 